\title{
PROX1 promotes human glioblastoma cell proliferation and invasion via activation of the nuclear factor- $\kappa B$ signaling pathway
}

\author{
$\mathrm{XUCHANG} \mathrm{XU}^{1}, \mathrm{XUEFENG} \mathrm{WAN}^{1}$ and XINTING WEI ${ }^{2}$ \\ ${ }^{1}$ Department of Neurosurgery, Zhumadian Central Hospital, Zhumadian, Henan 463000; ${ }^{2}$ Department of Neurosurgery, \\ The First Affiliated Hospital of Zhengzhou University, Zhengzhou, Henan 450000, P.R. China
}

Received November 7, 2015; Accepted November 8, 2016

DOI: $10.3892 / \mathrm{mmr} .2016 .6075$

\begin{abstract}
Prospero homeobox protein 1 (PROX1) is highly expressed in high-grade malignant astrocytic gliomas. However, the role of PROX1 in the pathogenesis of glioblastoma multiforme (GBM) remains unclear. The present study overexpressed PROX1 in human GBM cell lines and examined its effects on cell growth, tumorigenesis, and invasiveness. In addition, the involvement of the nuclear factor- $\mathrm{\kappa B}(\mathrm{NF}-\kappa \mathrm{B})$ signaling pathway in the action of PROX1 was examined. It was identified that overexpression of PROX1 significantly increased the proliferation and colony formation of glioblastoma cells, compared with empty vector-transfected controls. Furthermore, ectopic expression of PROX1 promoted the growth of GBM xenograft tumors. Western blot analysis revealed that PROX1 overexpression induced nuclear accumulation of NF-kB p65 and upregulated the expression levels of the NF- $\kappa \mathrm{B}$ responsive genes cyclin D1 and matrix metallopeptidase 9. An NF- $\kappa$ B reporter assay demonstrated that PROX1-overexpressing glioblastoma cells had significantly greater NF- $\kappa \mathrm{B}$-dependent reporter activities compared with empty vector-transfected controls. Transfection of a dominant inhibitor of $\kappa \mathrm{B} \alpha$ mutant into PROX1-overexpressing cells significantly impaired their proliferation and invasion capacities, which was accompanied by reduced levels of nuclear NF- $\kappa B$ p65. Collectively, these data indicated that PROX1 serves an oncogenic role in GBM and promotes cell proliferation and invasiveness potentially via activation of the NF- $\mathrm{KB}$ signaling pathway. Therefore, PROX1 may represent a potential target for the treatment of GBM.
\end{abstract}

\section{Introduction}

Glioblastoma multiforme (GBM) is the most common lethal type of brain cancer characterized by rapid growth and

Correspondence to: Dr Xuchang Xu, Department of Neurosurgery, Zhumadian Central Hospital, 747 Zhonghua Road, Zhumadian, Henan 463000, P.R. China

E-mail: 2074718959@qq.com

Key words: apoptosis, glioblastoma, invasion, prospero homeobox protein 1 , tumorigenesis extensive invasiveness (1). The prognosis of GBM is poor; it has been reported that the five-year survival rate is only $9.8 \%$ in GBM patients treated with a combination of temozolomide-based chemotherapy and radiotherapy (2). The median overall survival period is 19.6 months following treatment with concurrent adjuvant chemotherapy and radiotherapy (3). It is important to identify the key molecular factors contributing to the aggressive phenotype of glioblastoma cells to develop novel therapies for the treatment of this disease.

Prospero homeobox protein 1 (PROX1) is a transcription factor expressed in various tissues, including the heart, liver, skeletal muscles, pancreas, kidney and brain (4). This protein is critical for organ development during embryogenesis $(5,6)$. In addition, PROX1 is involved in tumorigenesis and progression. For example, PROX1 has been revealed to promote the survival of colon cancer cells under metabolic stress and facilitate the spread of tumor cells $(7,8)$. Similarly, overexpression of PROX1 enhances the migration and invasion of hepatocellular carcinoma (HCC) cells, and HCC metastasis in nude mice (9). However, ectopic expression of PROX1 exerts anti-proliferative effects against esophageal (10) and pancreatic (11) cancer cells. In high-grade malignant astrocytic gliomas, PROX1 is highly expressed (12). PROX1 has been identified as an independent prognostic factor for survival in patients with World Health Organization grade II gliomas (13). These findings implicate PROX1 in the pathogenesis of GBM.

The present study overexpressed PROX1 in human GBM cell lines and examined its roles in glioblastoma cell growth, tumorigenesis and invasiveness. As oncogenic nuclear factor- $\mathrm{KB}(\mathrm{NF}-\mathrm{\kappa B})$ signaling is critical for the growth and progression of GBM (14), whether the action of PROX1 was mediated through modulation of NF- $\mathrm{kB}$ activation was additionally investigated.

\section{Materials and methods}

Cell lines. The LN-229 and U87MG human glioma cell lines were purchased from the Cell Bank of the Chinese Academy of Sciences (Shanghai, China). They were cultured in Dulbecco's modified Eagle's medium (DMEM) supplemented with $10 \%$ fetal bovine serum (FBS), $100 \mathrm{U} / \mathrm{ml}$ penicillin and $100 \mu \mathrm{g} / \mathrm{ml}$ streptomycin (Invitrogen; Thermo Fisher Scientific, Inc., Waltham, MA, USA) at $37^{\circ} \mathrm{C}$ in a humidified atmosphere of $5 \% \mathrm{CO}_{2}$. 
Plasmids and transfection. Full-length human PROX1 cDNA (OriGene Technologies, Inc., Rockville, MD, USA) was cloned into the expression vector pcDNA3.1(+) (Invitrogen; Thermo Fisher Scientific, Inc.). The sequence identity of the pcDNA3.1/PROX1 construct was confirmed.

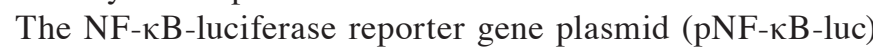
was purchased from Stratagene; Agilent Technologies, Inc. (Santa Clara, CA, USA) and the Renilla luciferase expression plasmid (pRL-TK) was purchased from Promega Corporation (Madison, WI, USA). A plasmid expressing dominant negative inhibitor of $\kappa \mathrm{B} \alpha(\mathrm{I} \kappa \mathrm{B} \alpha)$ containing serine to alanine mutations at positions 32 and 36 (pCMV-IкB $\alpha M$ ) was purchased from Clontech Laboratories, Inc. (Mountainview, CA, USA).

At $\sim 80 \%$ confluence, U87MG and LN-229 cells were transfected with $0.5 \mu \mathrm{g}$ pcDNA3.1(+) empty vector or pcDNA3.1/PROX1 using Lipofectamine ${ }^{\circledR} 2000$ (Invitrogen; Thermo Fisher Scientific, Inc.) according to the manufacturer's protocol. Selection was performed with G418 (Sigma-Aldrich; Merck Millipore, Darmstadt, Germany) at $800 \mu \mathrm{g} / \mathrm{ml}$ and resistant clones were obtained and pooled two weeks after transfection. PROX1 overexpression was confirmed by western blot analysis. For the NF- $\mathrm{kB}$ reporter assay, vector or PROX1 stably-transfected cells or parental cells were seeded in triplicate at $1 \times 10^{5}$ cells per well into 12 -well plates $24 \mathrm{~h}$ prior to transfection. Cells were co-transfected with $0.5 \mu \mathrm{g}$ pNF- $\kappa \mathrm{B}-$ luc together with $0.1 \mu \mathrm{g}$ pRL-TK. Luciferase activity was measured $24 \mathrm{~h}$ after transfection with the Dual-Luciferase Reporter assay system (Promega Corporation) according to the manufacturer's protocol. The relative luciferase activity was determined by normalization to Renilla luciferase activity. For inhibitory studies, vector or PROX1 stably-transfected cells were co-transfected with $0.5 \mu \mathrm{g} \mathrm{pCMV-I \kappa B} \alpha \mathrm{M}$ or pCMV plasmid. At $24 \mathrm{~h}$ post-transfection, cells were collected and subjected to gene expression analysis and cell proliferation and invasion assays.

Cell proliferation assay. Cell proliferation was determined using the 3-(4,5-dimethylthiazol-2-yl)-2,5-diphenyltetrazolium bromide (MTT) assay (Sigma-Aldrich; Merck Millipore). Briefly, U87MG and LN-229 cells $\left(3 \times 10^{3}\right)$ were seeded into 96-well plates and cultured for three days. MTT solution at a final concentration of $5 \mathrm{mg} / \mathrm{ml}$ was added to each well and incubated at $37^{\circ} \mathrm{C}$ for $4 \mathrm{~h}$. Dimethyl sulfoxide was added to dissolve formazan crystals. The number of viable cells was determined by measuring absorbance at a wavelength of $570 \mathrm{~nm}$ using a microplate reader.

Colony formation assay. For the colony formation assay, U87MG and LN-229 cells (600 per well) were plated into 6-well plates and cultured for 10 days in DMEM containing $10 \%$ FBS. Cells were fixed with methanol and stained with $0.1 \%$ crystal violet (Sigma-Aldrich; Merck Millipore). Individual colonies consisting of 50 or more cells were counted.

Xenograft studies. A total of 12 male BALB/c nude mice at 5 weeks of age were obtained from the Animal Center of Zhengzhou University (Zhengzhou, China) and housed at $24^{\circ} \mathrm{C}$ under a 12 -h light/dark cycle with free access to water and standard laboratory food. The experimental protocols involving animals were approved by the Institutional Animal
Care and Use Committee of Zhengzhou University. Vector and PROX1 stably-transfected U87MG cells $\left(2 \times 10^{6}\right)$ were injected subcutaneously into the right flank of mice ( $n=6$ per group). Tumor size was measured with calipers every seven days and the tumor volume was calculated. Animals were anesthetized 35 days after injection of tumor cells with intraperitoneal injection of ketamine $(60 \mathrm{mg} / \mathrm{kg})$ and xylazine $(6 \mathrm{mg} / \mathrm{kg})$, which were purchased from Shanghai First Biochemical Pharmaceutical Co., Ltd. (Shanghai, China). Xenograft tumors were extracted and imaged. Tumor volume and weight were measured.

Transwell invasion assay. Transfected and parental U87MG and LN-229 cells in serum-free DMEM were plated into the upper chamber of an insert ( $8-\mu \mathrm{m}$ pore size) precoated with Matrigel (BD Biosciences, Franklin Lakes, NJ, USA). The lower chamber was filled with media containing $10 \%$ FBS. Cells were allowed to invade Matrigel-coated inserts at $37^{\circ} \mathrm{C}$ for $24 \mathrm{~h}$. Subsequently, non-invaded cells were removed with a cotton swab. The cells that had invaded through the insert were stained with $0.1 \%$ crystal violet in methanol and imaged using an inverted light microscope. Cells in five random fields per insert were counted.

Western blot analysis. For the extraction of total cellular proteins, cells were washed with ice-cold phosphate-buffered saline and lysed with lysis buffer $(50 \mathrm{mM}$ 4-(2-hydroxyethyl)-1-piperazineethanesulphonic acid at, $\mathrm{pH} 7.5$, with $150 \mathrm{mM} \mathrm{NaCl}, 1 \mathrm{mM}$ EDTA, $10 \%$ glycerol and $1 \%$ Triton X-100) containing a Protease Inhibitor Cocktail (Sigma-Aldrich; Merck Millipore). Lysates were centrifuged at $12,000 \mathrm{x} g$ for $10 \mathrm{~min}$ at $4^{\circ} \mathrm{C}$ to remove debris, and the supernatants containing total proteins were collected for further analysis. Nuclear and cytoplasmic sub-fractions were prepared using a Nuclear/Cytosol Fractionation kit (BioVision, Inc., Milpitas, CA, USA) according to the manufacturer's protocol. Equal quantities of protein samples (50 $\mu \mathrm{g}$ per lane) were separated by 10-12\% SDS-polyacrylamide gel electrophoresis and transferred onto nitrocellulose membranes. Following the blocking of non-specific binding sites with 5\% non-fat milk, the membranes were incubated with primary antibodies overnight at $4^{\circ} \mathrm{C}$, followed by incubation with horseradish peroxidase-conjugated goat anti-mouse $\operatorname{IgG}$ (catalog no. sc-2005, Santa Cruz Biotechnology, Inc., Dallas, TX, USA; 1:2,000 dilution) or goat anti-rabbit IgG (catalog no. sc-2004, Santa Cruz Biotechnology, Inc.; 1:2,000 dilution) for $1 \mathrm{~h}$. The signal was detected using an Enhanced Chemiluminescence system (GE Healthcare Life Sciences, Chalfont, UK). The primary antibodies (all at 1:500 dilution) used were as follows: Rabbit polyclonal anti-PROX1 (catalog no. ab191019), rabbit monoclonal anti-cyclin D1 (catalog no. ab137875), rabbit monoclonal anti-matrix metallopeptidase (MMP)-9 (catalog no. ab76003), mouse monoclonal anti- $\beta$-actin (catalog no. ab184220) and rabbit monoclonal anti-NF-кB p65 (catalog no. ab76311) obtained from Abcam, Cambridge, MA, USA; and mouse monoclonal anti-histone $\mathrm{H} 3$ (catalog no. 14269), rabbit monoclonal anti-phosphorylated (p)-IкB $\alpha$ (catalog no. 2859), rabbit monoclonal anti-IкB $\alpha$ (catalog no. 4812), mouse monoclonal anti-p-protein kinase B (Akt; catalog no. 12694), mouse monoclonal anti-Akt (catalog no. 2920), 


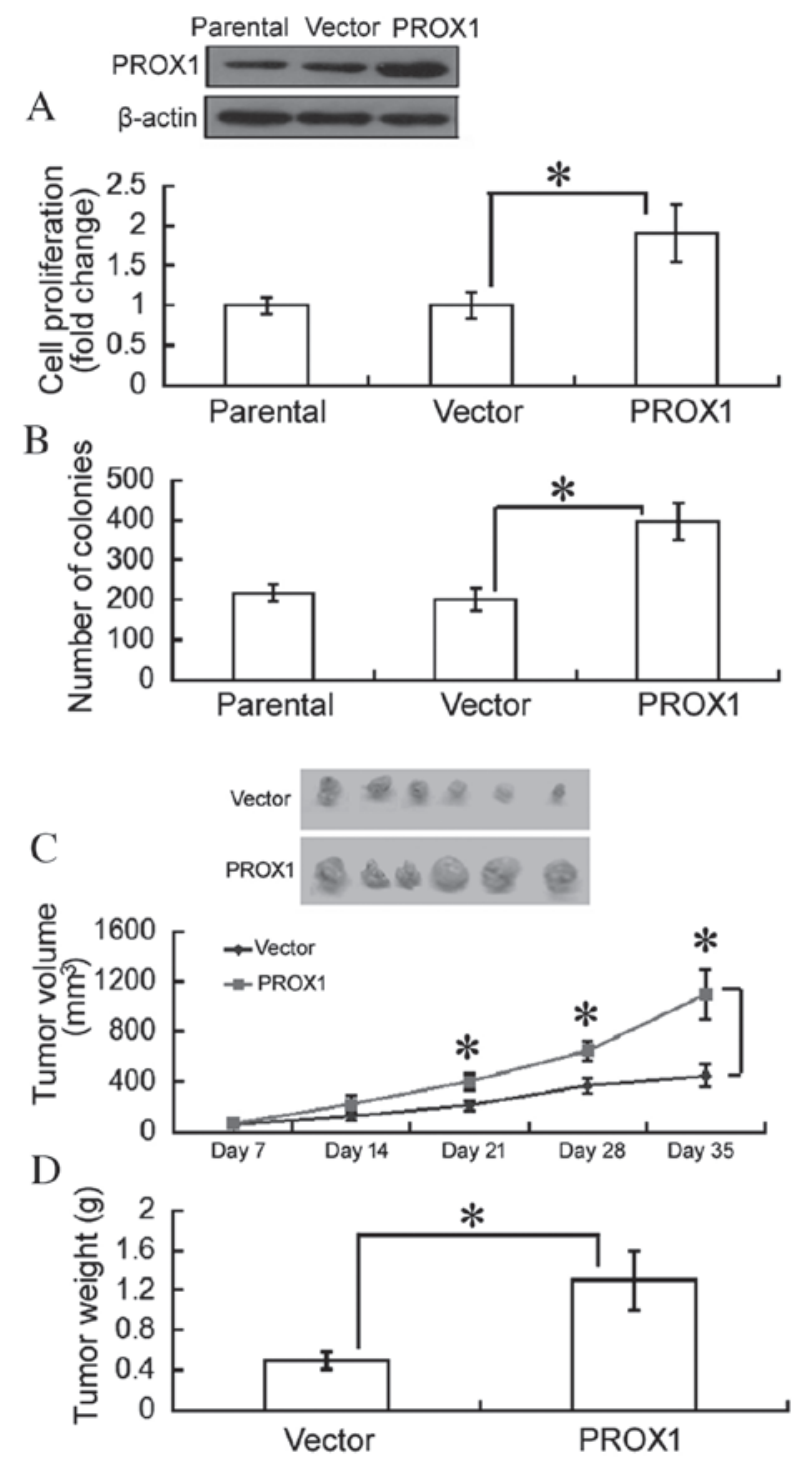

Figure 1. Overexpression of PROX1 promotes tumorigenesis of human glioblastoma cells. (A) Measurement of the proliferation of parental U87MG cells and those stably transfected with empty vector or PROX1-expressing plasmid, as assessed by an MTT assay following a 3-day culture. ${ }^{*} \mathrm{P}<0.05$. A representative western blot is presented to demonstrate successful PROX1 overexpression. (B) A colony formation assay was performed to examine the clonogenic growth of U87MG cells with or without PROX1 overexpression. ${ }^{*} \mathrm{P}<0.05$. U87MG cells stably transfected with pcDNA3.1(+) or pcDNA3.1/PROX1 were implanted into male BALB/c nude mice and tumor growth was assessed. (C) Tumor volume was measured every week following cell injection. ${ }^{*} \mathrm{P}<0.05$ vs. vector-transfected tumors at the same time-point. Representative tumor images are presented. (D) Animals were sacrificed at 35 days and tumors were extracted and weighed. ${ }^{*} \mathrm{P}<0.05$. PROX1, prospero homeobox protein 1 .

rabbit polyclonal anti-p-extracellular signal-regulated kinase (ERK; catalog no. 9101) and rabbit monoclonal anti-ERK (catalog no. 4695) purchased from Cell Signaling Technology, Inc., Danvers, MA, USA).

Statistical analysis. Data are presented as the mean \pm standard deviation. Statistical analyses were performed with SPSS version 16.0 software (SPSS, Inc., Chicago, IL, USA) using one-way analysis of variance followed by the Tukey post-hoc test. $\mathrm{P}<0.05$ was considered to indicate a statistically significant difference.

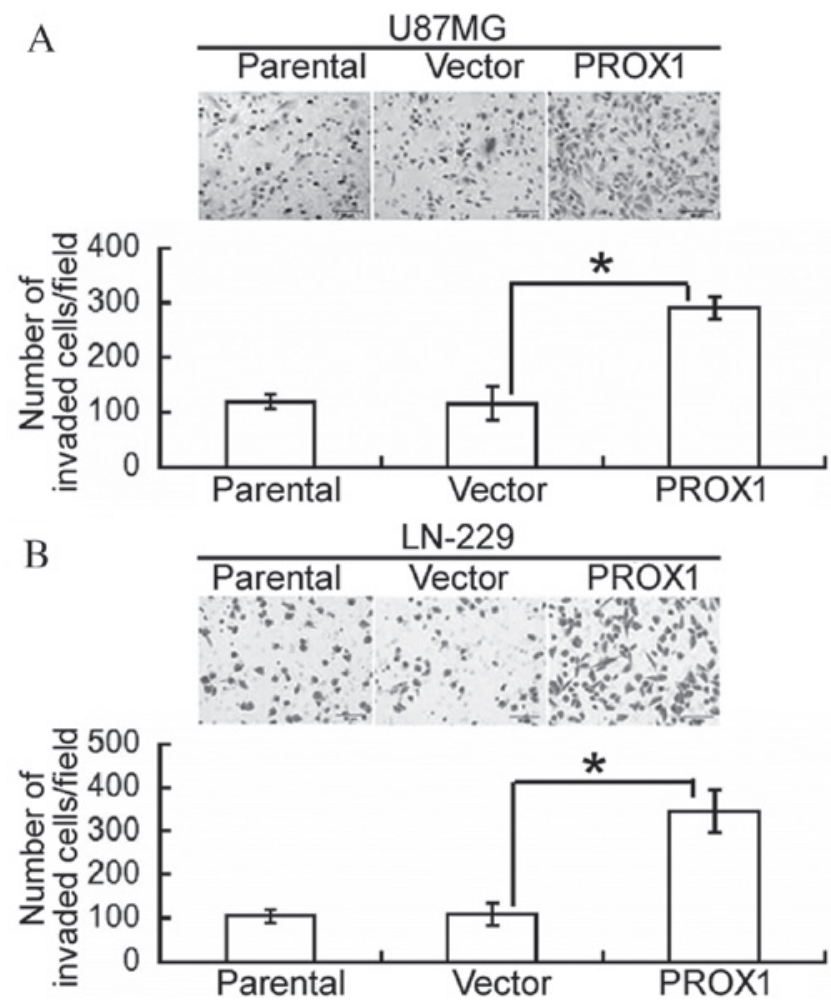

Figure 2. PROX1 overexpression increases glioblastoma cell invasion. (A) U87MG and (B) LN-229 cells were stably transfected with an empty vector or a PROX1-expressing plasmid and subjected to a Transwell invasion assay. Following incubation for $24 \mathrm{~h}$, the cells that had invaded through the insert were stained with crystal violet and counted. Results from three independent experiments are presented. ${ }^{*} \mathrm{P}<0.05$. PROX1, prospero homeobox protein 1 .

\section{Results}

Overexpression of PROX1 promotes tumorigenesis of human glioblastoma cells. To determine the role of PROX1 in glioblastoma cell growth, PROX1 was overexpressed in U87MG and LN-229 cells. An MTT assay revealed that overexpression of PROX1 significantly enhanced the proliferation of U87MG cells following a 3-day culture, compared with empty vector-transfected cells ( $\mathrm{P}=0.0342$; Fig. 1A). The numbers of colonies were significantly increased in PROX1-overexpressing U87MG cells, as assessed by colony formation assays $(\mathrm{P}=0.0206$; Fig. 1B). Similar results were observed in LN-229 cells (data not shown).

To further confirm the oncogenic role of PROX1 in vivo, tumor xenografts from vector- or PROX1-transfected U87MG cells were generated. Growth of PROX1 tumor xenografts was significantly increased compared with empty vector tumors $(\mathrm{P}<0.05$; Fig. 1C). At 35 days, tumor weight was significantly greater in the PROX1 group compared with the empty vector group $(\mathrm{P}=0.0379$; Fig. 1D).

PROX1 enhances glioblastoma cell invasion. Following this, the effect of PROX1 overexpression on the invasiveness of glioblastoma cells was assessed. Compared with vector-transfected U87MG cells, PROX1 overexpression significantly increased the number of invading cells following a 24-h incubation ( $\mathrm{P}=0.0074$; Fig. 2A). Similarly, 
A

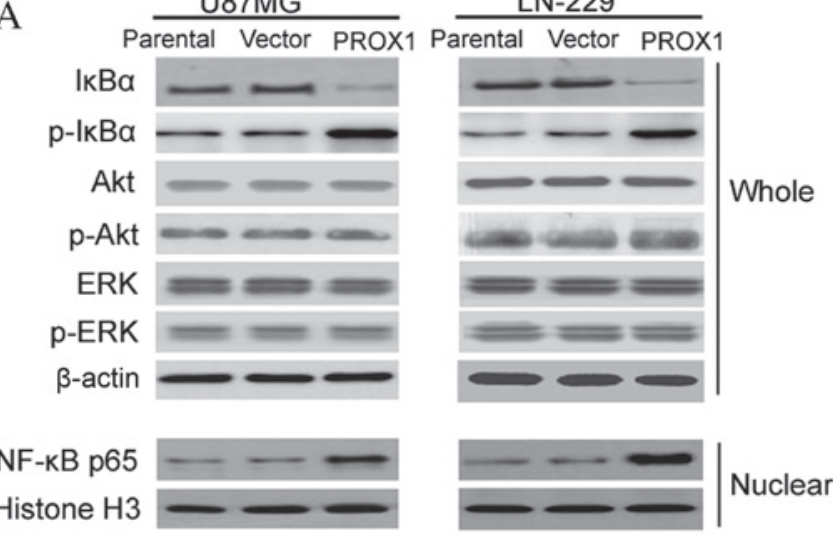

Figure 3. PROX1 overexpression induces sustained NF- $\kappa \mathrm{B}$ activation. (A) Western blot analysis of proteins in whole cellular lysates or nuclear extracts from U87MG and LN-229 cells with or without PROX1 overexpression. Representative blots of three independent experiments are presented. (B) Vector or PROX1 stably-transfected or parental cells were co-transfected with an NF- $\mathrm{kB}-$ luciferase reporter together with a pRL-TK control reporter. The relative luciferase activity was determined following normalization to Renilla luciferase activity. ${ }^{*} \mathrm{P}<0.05$. (C) Western blot analysis of the expression of cyclin D1 and MMP-9. Representative blots of three independent experiments are presented. PROX1, prospero homeobox protein 1; nuclear factor- $\kappa \mathrm{B}, \mathrm{NF}-\kappa \mathrm{B}$; MMP-9, matrix metallopeptidase-9; $\mathrm{p}$, phosphorylated; I $\mathrm{I} B \alpha$, inhibitor of $\kappa \mathrm{B} \alpha$; Akt, protein kinase B; ERK, extracellular signal-regulated kinase.

PROX1-overexpressing LN-229 cells demonstrated a significantly greater invasive capacity compared with control cells ( $\mathrm{P}=0.0125$; Fig. 2B).

PROXI overexpression induces sustained $N F-\kappa B$ activation . The involvement of signaling pathways in the oncogenic activity of PROX1 was subsequently investigated. Western blot analysis revealed that PROX1 overexpression induced the

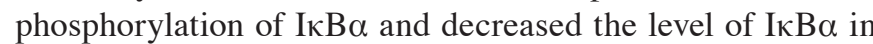
U87MG and LN-229 cells (Fig. 3A). Nuclear accumulation of NF- $\mathrm{B}$ p65 was detected in PROX1-overexpressing cells. However, the phosphorylation levels of Akt and ERK were not altered by PROX1 overexpression. To confirm the effect of PROX1 overexpression on NF- $\mathrm{KB}$ activation, vector or PROX1 stably-transfected U87MG and LN-229 cells were co-transfected with an NF- $\kappa B$-dependent reporter gene and a pRL-TK control vector. PROX1-overexpressing U87MG and LN-229 cells demonstrated a 3.5- and 5.8-fold increase

A

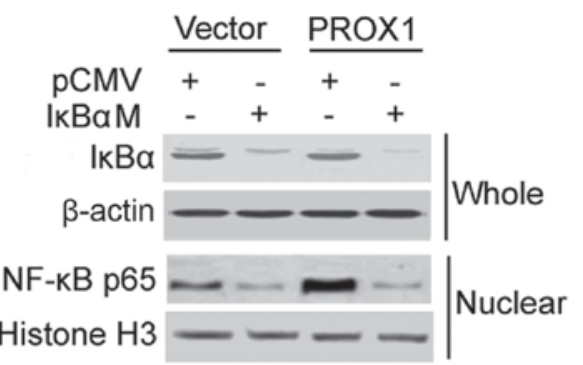

B

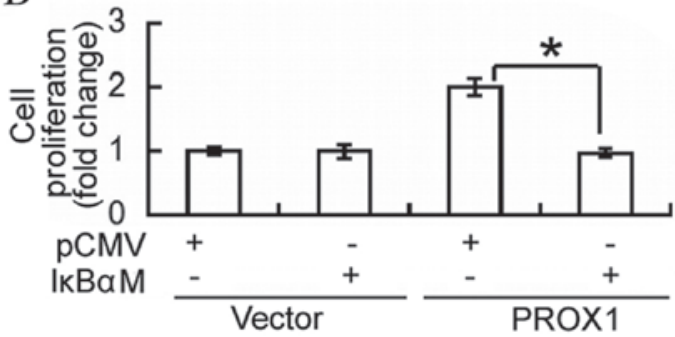

C

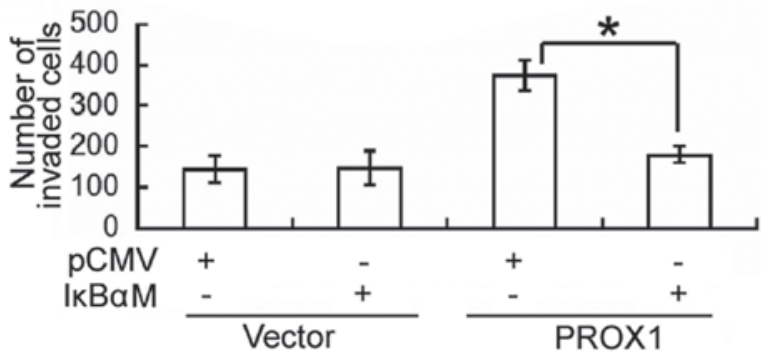

Figure 4. Inhibition of $\mathrm{NF}-\kappa \mathrm{B}$ activity attenuates the oncogenic activity of PROX1 in glioblastoma cells. (A) Vector or PROX1 stably-transfected cells were co-transfected with a pCMV-I $\mathrm{BB} \alpha \mathrm{M}$ or pCMV plasmid. At $24 \mathrm{~h}$ post-transfection, cells were collected for western blot analysis of $\mathrm{I} \kappa \mathrm{B} \alpha$

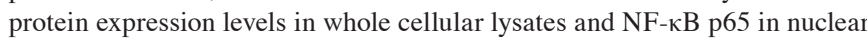
extracts. (B) Cells were transfected with the indicated constructs and cultured for 3 days prior to measurement of cell viability using an MTT assay. ${ }^{*} \mathrm{P}<0.05$ (C) A Transwell invasion assay was performed to assess the invasiveness of glioblastoma cells transfected with the indicated constructs. Results from three independent experiments are presented. ${ }^{*} \mathrm{P}<0.05$. PROX1, prospero homeobox protein 1 ; nuclear factor- $\kappa \mathrm{B}, \mathrm{NF}-\kappa \mathrm{B}$; I $\kappa \mathrm{B} \alpha$, inhibitor of $\kappa \mathrm{B} \alpha$.

in NF-кB-dependent reporter activity, respectively, compared with vector-transfected controls (Fig. 3B). The expression of various NF- $\kappa \mathrm{B}$ responsive genes, including cyclin D1 and MMP-9, was subsequently determined. Enforced expression of PROX1 resulted in a marked elevation in the protein expression levels of cyclin D1 and MMP-9, compared with the vector-transfected controls (Fig. 3C).

Inhibition of NF- $\kappa B$ activity attenuates the oncogenic activity of PROX1 in glioblastoma cells. To determine whether the oncogenic role of PROX1 in glioblastoma cells is mediated via activation of NF- $\kappa \mathrm{B}$, a dominant I $\mathrm{I} \mathrm{B} \alpha$ mutant was transfected into PROX1-overexpressing U87MG cells, and cell proliferation and invasion were assessed. Western blot analysis confirmed the overexpression of the I $\kappa \mathrm{B} \alpha$ mutant in I $\mathrm{B} \alpha \mathrm{M}$-transfected cells, which was accompanied by reduced nuclear accumulation of NF- $\kappa$ B p65 (Fig. 4A). Notably, the delivery of the negative I $\mathrm{I} B \alpha$ mutant significantly impaired the proliferation and invasiveness of PROX1-overexpressing cells $(\mathrm{P}<0.0001$ and $\mathrm{P}=0.0042$, respectively; Fig. $4 \mathrm{~B}$ and $\mathrm{C})$. 
These results support an important role for $\mathrm{NF}-\kappa \mathrm{B}$ activation in PROX1-mediated induction of cell proliferation and invasion.

\section{Discussion}

PROX1 acts as a tumor suppressor or tumor promoter in different types of cancers. For example, in HCC PROX1 facilitates cancer cell invasion and metastasis (9), whereas in pancreatic cancer cells this protein induces growth-suppressive effects (1). Although previous studies have documented the high expression of PROX1 in GBM $(12,13)$, the biological functions of PROX1 in the tumorigenesis and invasiveness of glioblastoma cells remain unclear. The results of the present study revealed that overexpression of PROX1 significantly increased the proliferation and colony formation of GBM cells. In vivo studies further confirmed that PROX1 overexpression enhanced tumor xenograft growth. These results provide, to the best of our knowledge, the first evidence for the tumor-promoting role of PROX1 in GBM.

Glioblastoma cells possess a high invasiveness potential, which is an important factor contributing to poor prognosis of GBM $(15,16)$. Therefore, the effect of the overexpression of PROX1 on the invasiveness of glioblastoma cells was examined. An in vitro Transwell assay revealed that enforced expression levels of PROX1 significantly increased invasiveness through the Matrigel layer in U87MG and LN-229 cells. The pro-invasive activity of PROX1 in glioblastoma cells may provide an explanation for the significant association between high expression levels of PROX1 and poor prognosis in patients with grade II gliomas (13). In addition, PROX1 demonstrates the ability to modulate cell invasion in various other types of cancer cells. For example, ectopic expression of PROX1 has been revealed to promote colon cancer cell invasion via induction of epithelial-mesenchymal transition (17).

To determine the underlying mechanism of the tumor-promoting activities of PROX1, the effect of PROX1 overexpression on $\mathrm{NF}-\kappa \mathrm{B}$ activation was investigated. It was identified that PROX1-overexpressing cells had increased activation of $\mathrm{NF}-\kappa \mathrm{B}$, as evidenced by reduced $\mathrm{I} \kappa \mathrm{B} \alpha$ levels and nuclear accumulation of $\mathrm{NF}-\kappa \mathrm{B}$ p 65. Additionally, there was a significant increase in $\mathrm{NF}-\kappa \mathrm{B}$-dependent transcriptional activity in PROX1-overexpressing cells. Cyclin D1 and MMP-9 are two downstream genes of NF- $\kappa \mathrm{B}$ and serve important roles in tumor growth and progression $(18,19)$. Accompanying the activation of $\mathrm{NF}-\kappa \mathrm{B}, \mathrm{PROX} 1$ overexpression increased the protein expression levels of cyclin D1 and MMP-9 in glioblastoma cells. To confirm the involvement of $\mathrm{NF}-\kappa \mathrm{B}$ signaling in the action of PROX1, a dominant I $\kappa \mathrm{B} \alpha$ mutant was transfected into PROX1-overexpressing U87MG cells. Notably, overexpression of the I $\kappa \mathrm{B} \alpha$ mutant interfered with $N F-\kappa B$ activation and hindered the proliferation and invasiveness of PROX1-overexpressing cells. Taken together, these results suggested that the oncogenic role of PROX1 in glioblastoma cells may be at least partially mediated through the activation of the $\mathrm{NF}-\kappa \mathrm{B}$ signaling pathway. PROX1 has been demonstrated to promote HCC metastasis via upregulation of hypoxia-inducible factor $1 \alpha$ (9). Therefore, other signaling pathways may additionally mediate the action of PROX1 in glioblastoma cells.
The present study has various limitations. Firstly, overexpression studies do not fully address the pathophysiological roles of PROX1 in GBM. Knockdown experiments may confirm the requirement for PROX1 in the growth and progression of GBM. Secondly, there is a lack of information surrounding the association between PROX1 expression and $\mathrm{NF}-\kappa \mathrm{B}$ activation in GBM patients. Finally, the underlying mechanism of the regulation of $\mathrm{NF}-\kappa \mathrm{B}$ activation by PROX1 in glioblastoma cells remains to be fully elucidated.

In conclusion, the results of the present study demonstrated an oncogenic role for PROX1 in GBM. Overexpression of PROX1 was revealed to promote the tumorigenesis and invasiveness of glioblastoma cells, which was primarily associated with activation of the NF- $\kappa \mathrm{B}$ signaling pathway. PROX1 may represent a potential therapeutic target for the treatment of GBM; however, additional studies are required to confirm the impact of PROX1 in the pathogenesis of GBM.

\section{References}

1. Fukushima T, Tezuka T, Shimomura T, Nakano S and Kataoka H: Silencing of insulin-like growth factor-binding protein-2 in human glioblastoma cells reduces both invasiveness and expression of progression-associated gene CD24. J Biol Chem 282: 18634-18644, 2007.

2. Stupp R, Hegi ME, Mason WP, van den Bent MJ, Taphoorn MJ, Janzer RC, Ludwin SK, Allgeier A, Fisher B, Belanger K, et al: Effects of radiotherapy with concomitant and adjuvant temozolomide versus radiotherapy alone on survival in glioblastoma in a randomised phase III study: 5-year analysis of the EORTC-NCIC trial. Lancet Oncol 10: 459-466, 2009.

3. Lai A, Tran A, Nghiemphu PL, Pope WB, Solis OE, Selch M, Filka E, Yong WH, Mischel PS, Liau LM, et al: Phase II study of bevacizumab plus temozolomide during and after radiation therapy for patients with newly diagnosed glioblastoma multiforme. J Clin Oncol 29: 142-148, 2011.

4. Zinovieva RD, Duncan MK, Johnson TR, Torres R, Polymeropoulos $\mathrm{MH}$ and Tomarev SI: Structure and chromosomal localization of the human homeobox gene Prox 1. Genomics 35: 517-522, 1996.

5. Kim H, Cruz M, Bourdeau A and Dumont DJ: Cell-cell interactions influence vascular reprogramming by Prox 1 during embryonic development. PLoS One 8: e52197, 2013.

6. Sosa-Pineda B, Wigle JT and Oliver G: Hepatocyte migration during liver development requires Prox1. Nat Genet 25: 254-255, 2000 .

7. Ragusa S, Cheng J, Ivanov KI, Zangger N, Ceteci F, BernierLatmani J, Milatos S, Joseph JM, Tercier S, Bouzourene H, et al: PROX1 promotes metabolic adaptation and fuels outgrowth of Wnt(high) metastatic colon cancer cells. Cell Rep 8: 1957-1973, 2014.

8. Wiener Z, Högström J, Hyvönen V, Band AM, Kallio P, Holopainen T, Dufva O, Haglund C, Kruuna O, Oliver G, et al: Prox 1 promotes expansion of the colorectal cancer stem cell population to fuel tumor growth and ischemia resistance. Cell Rep 8: 1943-1956, 2014.

9. Liu Y, Zhang JB, Qin Y, Wang W, Wei L, Teng Y, Guo L, Zhang B, Lin Z, Liu J, et al: PROX1 promotes hepatocellular carcinoma metastasis by way of up-regulating hypoxia-inducible factor $1 \alpha$ expression and protein stability. Hepatology 58: 692-705, 2013.

10. Akagami M, Kawada K, Kubo H, Kawada M, Takahashi M, Kaganoi J, Kato S, Itami A, Shimada Y, Watanabe G and Sakai Y: Transcriptional factor Prox1 plays an essential role in the antiproliferative action of interferon- $\gamma$ in esophageal cancer cells. Ann Surg Oncol 18: 3868-3877, 2011.

11. Takahashi M, Yoshimoto T, Shimoda M, Kono T, Koizumi M, Yazumi S, Shimada Y, Doi R, Chiba T and Kubo H: Loss of function of the candidate tumor suppressor prox1 by RNA mutation in human cancer cells. Neoplasia 8: 1003-1010, 2006.

12. Elsir T, Eriksson A, Orrego A, Lindström MS and Nistér M: Expression of PROX1 is a common feature of high-grade malignant astrocytic gliomas. J Neuropathol Exp Neurol 69: 129-138, 2010. 
13. ElsirT,Qu M,Berntsson SG, Orrego A,Olofsson T,Lindström MS Nistér M, von Deimling A, Hartmann C, Ribom D and Smits A: PROX1 is a predictor of survival for gliomas WHO grade II. Br J Cancer 104: 1747-1754, 2011

14. Cherry EM, Lee DW, Jung JU and Sitcheran R: Tumor necrosis factor-like weak inducer of apoptosis (TWEAK) promotes glioma cell invasion through induction of NF- $\kappa \mathrm{B}$-inducing kinase (NIK) and noncanonical NF- $\kappa$ B signaling. Mol Cancer 14: 9, 2015.

15. Han S, Xia J, Qin X, Han S and Wu A: Phosphorylated SATB1 is associated with the progression and prognosis of glioma. Cell Death Dis 4: e901, 2013.

16. Siebzehnrubl FA, Silver DJ, Tugertimur B, Deleyrolle LP, Siebzehnrubl D, Sarkisian MR, Devers KG, Yachnis AT, Kupper MD, Neal D, et al: The ZEB1 pathway links glioblastoma initiation, invasion and chemoresistance. EMBO Mol Med 5: $1196-1212,2013$
17. Lu MH, Huang CC, Pan MR, Chen HH and Hung WC: Prospero homeobox 1 promotes epithelial-mesenchymal transition in colon cancer cells by inhibiting E-cadherin via miR-9. Clin Cancer Res 18: 6416-6425, 2012.

18. Bera A, Ghosh-Choudhury N, Dey N, Das F, Kasinath BS, Abboud HE and Choudhury GG: NFKB-mediated cyclin D1 expression by microRNA-21 influences renal cancer cell proliferation. Cell Signal 25: 2575-2586, 2013.

19. Lee GR, Jang SH, Kim CJ, Kim AR, Yoon DJ, Park NH and Han IS: Capsaicin suppresses the migration of cholangiocarcinoma cells by down-regulating matrix metalloproteinase- 9 expression via the AMPK-NF- $\kappa$ B signaling pathway. Clin Exp Metastasis 31: 897-907, 2014 